\title{
Identification of genes and molecular pathways involved in a C. elegans model of neuroborreliosis
}

$\frac{\text { Ruben Prado }{ }^{1,2}}{\text { Richard Nass }^{1,2}}$, Gai-Linn Bessing ${ }^{1}$, Nathaniel Snyder ${ }^{1}$, Gurpalik Singh ${ }^{3}$, Frank Yang ${ }^{3}$,

1Department of Pharmacology and Toxicology and Stark Neuroscience Research Institute, ${ }^{2}$ Indiana Clinical and Translational Sciences Institute, ${ }^{3}$ Department of Microbiology and Immunology, Indiana University School of Medicine, Indianapolis, IN

Background and Hypothesis: Lyme disease is caused by the spirochaete bacteria from the Borrelia species. Recent studies suggest that Lyme disease may be associated with dementia, brain atrophy, and protein aggregates that may be associated with the development of neurodegenerative diseases such as Parkinson's disease (PD) and Alzheimers disease (AD). The molecular basis of the Borrelia-associated innate immune response and associated neuropathology is poorly defined. A significant hindrance in dissecting these molecular components is the lack of facile in vivo genetic models to explore the mechanisms involved in the neuropathology. Here we hypothesize that the nematode $C$. elegans will be a useful model for Borrelia-associated innate immunity and neuropathology.

Project Methods: We utilized transcriptional reporters, transgenic animals, neuronal morphology analysis, RNAi, host defense pathways, AD- and PD-associated pathologies, and behavior assays to determine the effect that Borrelia has on C. elegans viability.

Results: C. elegans can be infected and survive using Borrelia as a food source, and the bacteria can induce highly conserved innate immune response pathways, and exacerbate PD-associated dopamine neuronal death in human A53T $\alpha$-synuclein-expressing animals. C. elegans models expressing AD-associated human $A \beta \quad 1-42$ also show significant movement defects and increased protein aggregates when exposed to Borrelia.

Conclusions and Potential Impact: This study further characterizes a novel genetic model for Borrelia-associated innate immunity and neuropathology. Incorporating C. elegans genetic screens, this model should allow us to identify mediators of the Borreliaassociated pathologies that should facilitate the identification of molecular pathways and potential therapeutic targets. 